\title{
The cardioprotective effect of interleukin-11 against ischemia- reperfusion injury in a heart donor model
}

\author{
Yusaku Tamura $^{1}$, Hiroki Kohno ${ }^{1}$, Tomomi Mohri ${ }^{2}$, Yasushi Fujio ${ }^{2}$, Goro Matsumiya ${ }^{1}$ \\ ${ }^{1}$ Department of Cardiovascular Surgery, Graduate School of Medicine, Chiba University, Chiba, Japan; ${ }^{2}$ Laboratory of Clinical Science and \\ Biomedicine, Graduate School of Pharmaceutical Sciences, Osaka University, Osaka, Japan \\ Correspondence to: Goro Matsumiya, MD, PhD. Department of Cardiovascular Surgery, Graduate School of Medicine, Chiba University, 1-8-1 \\ Inohana, Chuo-ku, Chiba, 260-8677, Japan. Email: matsumg@faculty.chiba-u.jp.
}

\begin{abstract}
Background: Previously, we have demonstrated the cardioprotective effect of interleukin (IL)-11 in animal models of acute coronary syndrome. In this study, we sought to evaluate its cardioprotective potential during prolonged hypothermic global ischemia and subsequent reperfusion using a rat heart donor model.

Methods: IL-11 was administered intravenously 10 minutes before harvesting the rat heart. The hearts were preserved in cold $\left(4^{\circ} \mathrm{C}\right)$ Krebs-Henseleit buffer for 6 hours, and then attached to a Langendorff perfusion apparatus and reperfused with an oxygenated Krebs-Henseleit solution containing IL-11. Normal saline was used instead of IL-11 in the control group. Functional recovery of the reperfused heart was observed by using a left ventricular balloon. Myocardial cell injury was quantified by measuring the biomarkers collected from the coronary effluent. Apoptotic cells were identified and counted using the terminal deoxynucleotide transferase-mediated dUTP nick-end labeling (TUNEL) staining technique.

Results: IL-11 administration improved myocardial function after 6 hours of cold ischemia. Although there were no significant differences in any of the baseline-measured values between the two groups, left ventricular developed pressure (LVDP) and changes in left ventricular pressures (dP/dt) were significantly higher in the IL-11 group at 120-minute reperfusion. The number of TUNEL-labeled cardiomyocytes was also significantly smaller in the IL-11 group.

Conclusions: The administration of IL-11 showed a significant recovery of cardiac contractile function after 6 hours of cold ischemia. Our data suggest that it may have significant therapeutic potential for maintaining the functional viability of the heart exposed to prolonged hypothermic global ischemia.
\end{abstract}

Keywords: Animal model; inflammatory mediators; ischemia/reperfusion injury (heart); organ preservation; transplantation (heart)

Submitted Jun 23, 2017. Accepted for publication Sep 04, 2017.

doi: 10.21037/acs.2017.09.11

View this article at: http://dx.doi.org/10.21037/acs.2017.09.11

\section{Introduction}

Heart failure is a leading cause of mortality worldwide, and for patients who remain symptomatic despite maximum medical therapy, heart transplantation is the final most effective treatment available. However, the enlarging disparity between the supply and demand for donor hearts has greatly limited this treatment, prompting researchers to find alternatives to the treatment or ways to increase organ supply. Another major limitation to heart transplantation is organ preservation. The universally practiced heart preservation technique using cold ischemic storage is largely dependent on time; cold ischemic duration of longer than 4 hours has been shown to aggravate ischemia-reperfusion (IR) injury and adversely affect survival (1). Therefore, developing a preservation method that offers a longer tolerable ischemic time may not only improve outcome but facilitate distant procurement and ultimately increase the overall donor pool.

Interleukin (IL)-11 is a hematopoietic IL-6 family 
cytokine with pleiotropic properties (2) that may have a cardioprotective role against IR insults. Although the underlying mechanism remains unclear, evidence from previous works has suggested that IL-11 enhances cellular protection and healing. Recently, we found that cardiomyocytes express a cell surface IL-11 receptor bound to a cytokine-specific transmembrane glycoprotein (gp130) and showed in a murine IR injury model that pretreatment with IL-11 provides protection against IR injury through a preconditioning effect (3). We also demonstrated that IL-11 reduces adverse cardiac remodeling, possesses antiapoptotic features, promotes angiogenesis after myocardial infarction (4), and exhibits a protective post-conditioning effect against IR injury through a signal transducer and activator of transcription protein (STAT3) activation (5). These results suggest that IL-11 may have a potential role in maintaining organ viability of the donor heart subjected to prolonged ischemia. In this study, we sought to examine this cardioprotective effect of IL-11 using a rat donor heart model.

\section{Methods}

Between July and November of 2013 and following the approval of the institutional committee, 20 male SpragueDawley rats weighing approximately $300 \mathrm{~g}$ were used for this study. The rats were anesthetized with intraperitoneal medetomidine $(0.3 \mathrm{mg} / \mathrm{kg})$ and butorphanol tartrate $(1 \mathrm{mg} / \mathrm{kg})$, and heparinized (300 IU/kg) before intubation and mechanical ventilation. Sternotomy was then performed, and the heart from each rat was removed and quickly placed in ice-cold Krebs-Henseleit (K-H) buffer solution to promote rapid arrest. Residual blood in the coronary arteries was gently washed out with the cold buffer solution antegradely through the ascending aorta. The isolated heart was preserved in cold $\left(4^{\circ} \mathrm{C}\right) \mathrm{K}-\mathrm{H}$ buffer solution for a scheduled duration and then attached to a modified Langendorff perfusion apparatus by cannulating the ascending aorta. The heart was reperfused with an oxygenated $\mathrm{K}-\mathrm{H}$ perfusion fluid (in mmol/L; $118 \mathrm{NaCl}$, $25 \mathrm{NaHCO}_{3}, 4.7 \mathrm{KCl}, 1.2 \mathrm{KH}_{2} \mathrm{PO}_{4}, 1.2 \mathrm{MgCl}_{2}, 2.5 \mathrm{CaCl}_{2}$, 0.5 ethylenediaminetetraacetic acid, and 11 glucose) through this cannula at a hydrostatic pressure of $100 \mathrm{mmHg}$. This perfusion fluid was passed through a $0.45-\mu \mathrm{m}$ membrane filter (Sterivex-HV filter; Millipore) and gassed with 95\% oxygen and $5 \%$ carbon dioxide at $37{ }^{\circ} \mathrm{C}$ to achieve a $\mathrm{pH}$ of 7.4. The perfusion fluid was maintained at $37^{\circ} \mathrm{C}$ using a built-in heater, and the heart was placed inside a water- jacketed organ chamber, also maintained at $37^{\circ} \mathrm{C}$. The heart was reperfused under this condition for 2 hours. During reperfusion, a latex balloon (LB-2, Primetech Corporation, Tokyo, Japan) attached to the end of a piece of stiff polyethylene tubing was inserted into the left ventricle through the mitral valve. The balloon and the tubing were connected to a pressure transducer (MP5200, Edwards Lifesciences, Irvine, CA, USA) and filled with normal saline to produce a left ventricular end-diastolic pressure (LVEDP) of $0-10 \mathrm{mmHg}$ at baseline. The volume of the balloon was maintained constant throughout the experiment. LVEDP, left ventricular peak systolic pressure (LVSP), the peak rates of positive and negative changes in left ventricular pressure $(+\mathrm{dP} / \mathrm{dt}$ and $-\mathrm{dP} / \mathrm{dt}$, respectively), and heart rate were measured with a pressure amplifier (AP-641G Blood Pressure Amplifier, Nihon Kohden, Japan). Hemodynamic data and perfusion pressures were recorded continuously on a personal computer using PowerLab software (ML840, ADInstruments, Colorado Springs, CO, USA).

\section{Experimental protocol}

Recombinant human IL-11 was purchased from Peprotech EC (10 $\mu \mathrm{g}$ per ampule). In the IL-11 group $(\mathrm{n}=10)$, $12 \mu \mathrm{g} / \mathrm{kg}$ of recombinant human IL-11 was administered intravenously through the inferior vena cava 10 minutes before the heart was isolated. After 6 hours of cold ischemia, the heart was reperfused continuously at a constant pressure for 2 hours with an oxygenated normothermic K-H perfusion fluid containing $18 \mu \mathrm{g} / \mathrm{kg}$ of IL-11, which was added to the $\mathrm{K}-\mathrm{H}$ fluid reservoir.

In the control group $(n=10)$, similar doses of normal saline were administered before the procurement of the organ. During reperfusion, only the K-H fluid was allowed to perfuse through the heart.

\section{Cold ischemic time}

To reduce the number of rats sacrificed for this study, a preliminary study was conducted to determine the optimum cold ischemic time for comparing the two groups. Cold ischemic time is defined as the time from when the heart was taken out of the chest and immediately immersed in ice-cold buffer solution to the time it was reperfused with the warm fluid. Cold ischemic times of less than 4 hours showed good recovery in all hearts in the control group, whereas after 12 hours, none recovered. Two-thirds of the rat hearts in the control group recovered at 6 hours in 
the preliminary study. This ischemic time (6 hours) was therefore used for treatment evaluation.

\section{Hemodynamic parameters and cardiac biomarkers}

Cardiac contractile strength was determined by calculating the maximum left ventricular developed pressure (LVDP) and by measuring LVSP, LVEDP, $+\mathrm{dP} / \mathrm{dt}$, and $-\mathrm{dP} / \mathrm{dt}$. These parameters were recorded using a pressure transducer connected to the balloon. LVDP was calculated as the difference between LVSP and LVEDP.

Coronary effluents from the heart were collected at 30 (baseline), 60, 90, and 120 minutes of reperfusion. Creatine kinase-MB (CK-MB) and aspartate aminotransferase (AST) were measured from the collected effluent by spectrophotometry.

\section{TTC staining and measurement of the necrotic area}

At the end of each experiment, the heart was trimmed and sliced transversely at the level of the papillary muscles. The slices were immersed in phosphate-buffered saline containing $2 \%$ triphenyltetrazolium chloride (TTC) for 15 minutes at $37^{\circ} \mathrm{C}$. Immediately after staining, photographs of the slices were taken with a digital camera for subsequent measurement of the necrotic area.

\section{TUNEL assay}

After the 120-minute of reperfusion and observation, 15 sections from each heart were prepared by slicing the frozen heart specimen transversely at the level of the papillary muscles and continuing down to the apex of the heart. A total of 75 sections in each group were prepared for the assay ( 5 hearts from the IL-11 group and 5 hearts from the control group). Apoptotic cells were identified and counted by using the terminal deoxynucleotide transferase-mediated dUTP nick-end labeling (TUNEL) staining technique and a detection kit (Apoptosis Detection Kit, TaKaRa BIO INC, Japan).

\section{Statistical analysis}

All numerical data are expressed as mean \pm standard deviation. The comparison between two groups was performed using an unpaired $t$-test. Differences were considered statistically significant when the calculated $\mathrm{P}$ value was less than 0.05 .

\section{Results}

\section{Hemodynamic parameters}

There were no significant differences in the baselinemeasured values between the two groups (LVDP: $25.5 \pm 11.7$ vs. $31.1 \pm 14.0, \mathrm{P}=0.34 ;+\mathrm{dP} / \mathrm{dt}: 611 \pm 293$ vs. $772 \pm 433, \mathrm{P}=0.34$; $-\mathrm{dP} / \mathrm{dt}:-327 \pm 152$ vs. $-407 \pm 235, \mathrm{P}=0.38$; control vs. IL-11, respectively). However, at 60 minutes of reperfusion, LVDP was significantly higher in the IL-11 group $(29.5 \pm 15.5 \mathrm{vs}$. $45.4 \pm 17.9, \mathrm{P}=0.048)$. At 90 minutes of reperfusion, both LVDP and $+\mathrm{dP} / \mathrm{dt}$ were significantly higher in the IL-11 group (LVDP: $29.6 \pm 17.4 v s .48 .8 \pm 18.7, \mathrm{P}=0.03 ;+\mathrm{dP} / \mathrm{dt}$ : $737 \pm 475$ vs. $1,256 \pm 604, \mathrm{P}=0.046)$. At 120 minutes of reperfusion, all parameters were significantly higher in the IL-11 group (LVDP: $30.0 \pm 17.4$ vs. $48.2 \pm 16.7, \mathrm{P}=0.03 ;+\mathrm{dP} /$ dt: $706 \pm 478$ vs. $1,240 \pm 441, \mathrm{P}=0.02 ;-\mathrm{dP} / \mathrm{dt}:-395 \pm 226$ vs. $-659 \pm 228, \mathrm{P}=0.02)$ (Figure 1).

\section{Myocardial biomarkers}

There were no significant differences in CK-MB and AST measured at each reperfusion interval between the two groups (Figure 2).

\section{TTC staining}

No necrosis was detected by TTC in both groups.

\section{TUNEL assay}

The number of TUNEL-labeled cardiomyocytes was significantly smaller in the IL-11 group than the control group (Figure 3).

\section{Discussion}

To our knowledge, this is the first study that has assessed the efficacy of IL-11 in a setting that simulates cardiac donor ischemia and reperfusion. The main findings were: (I) IL-11 significantly ameliorated the functional recovery of the heart subjected to prolonged cold ischemia followed by normothermic reperfusion; and (II) IL-11 reduced the number of apoptotic cardiomyocytes. The clinical implication of these findings is that IL-11 may have a therapeutic role in alleviating cardiac impairment often seen after an extended period of ischemia induced during a complex cardiac operation or after donor heart procurement. 


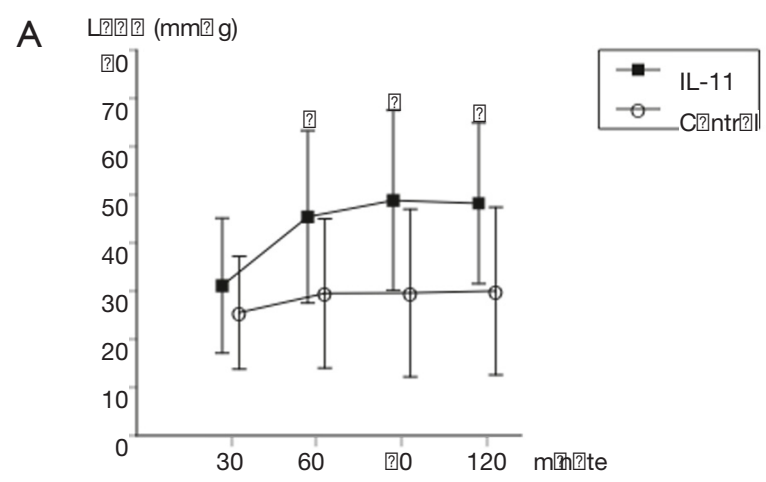

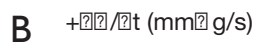
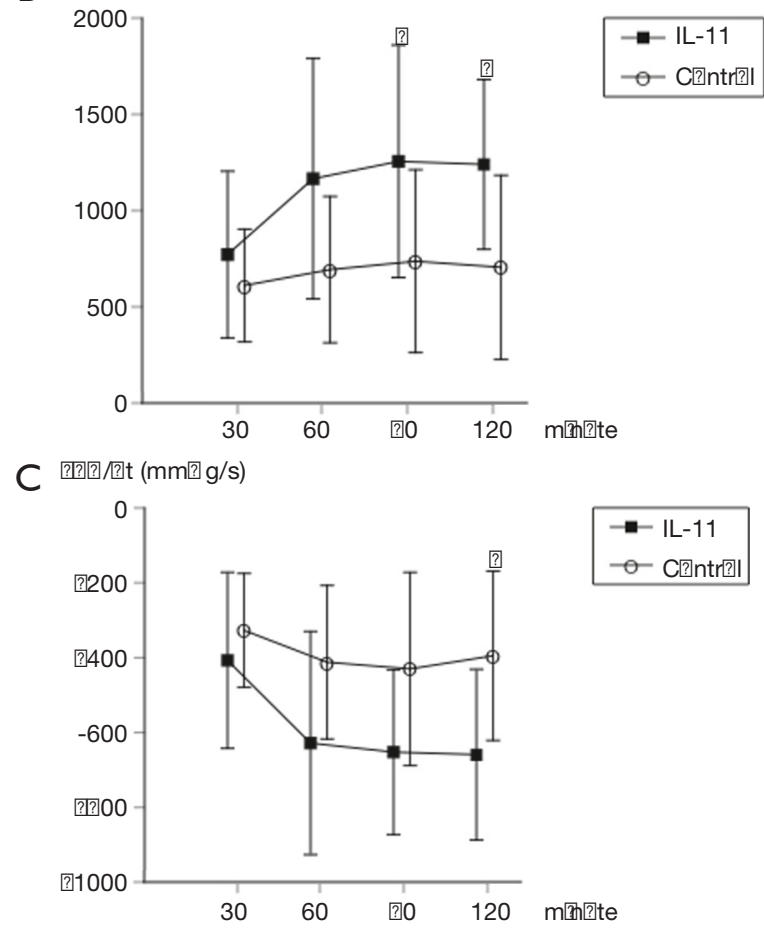

Figure 1 Comparison of hemodynamic parameters. IL-11 improved the left ventricular functional recovery of the heart subjected to prolonged cold ischemia during normothermic reperfusion, although no significant difference was observed in any of the values at 30 minutes of reperfusion compared to the control group. (A) LVDP was found to be significantly higher in the IL-11 group at 60, 90 and 120 minutes of reperfusion $(\mathrm{P}=0.048,0.03$ and 0.03 , respectively); (B) $+\mathrm{dP} / \mathrm{dt}$ was significantly higher in the IL-11 group at 90 and 120 minutes of reperfusion $(\mathrm{P}=0.046$ and 0.02 , respectively); (C) $-\mathrm{dP} / \mathrm{dt}$ was significantly higher in the IL-11 group at 120 minutes of reperfusion $(\mathrm{P}=0.02)$. Data are shown as mean \pm standard deviation. The comparison between both groups was performed using an unpaired $t$-test. ${ }^{*}, \mathrm{P}<0.05$ by unpaired $t$-test. IL-11, interleukin-11; LVDP, left ventricular developed pressure; $+\mathrm{dP} / \mathrm{dt}$, peak positive rate of left ventricular pressure rise; $-\mathrm{dP} / \mathrm{dt}$, peak negative rate of left ventricular pressure rise.
IL-11 is a multifunctional cytokine belonging to the IL-6 cytokine family and is known to exert pleiotropic effects on various organs, including brain, bone, testis, and intestines. Yet, to date, the physiologic role of this protein remains uncertain. Experimentally, IL-11 has been demonstrated to stimulate myelopoiesis, lymphopoiesis, erythropoiesis, and thrombopoiesis (6). It also enhances antigen-specific antibody responses, increases the plasma concentration of acute-phase proteins, and inhibits lipoprotein lipase activity (7). Clinically, owing to its thrombopoiesis effect, recombinant human $\mathrm{IL}-11$ is now used for the treatment of thrombocytopenia $(8,9)$. IL-11 also participates in the regulation of bone cell proliferation and differentiation (10) and may have potential as a therapy for osteoporosis. In addition, IL-11 exhibits an anti-inflammatory property and may be applied to the treatment of various chronic inflammatory diseases, such as Crohn's disease and rheumatoid arthritis (11). Furthermore, IL-11 appears to protect the intestinal mucosa during oxidant stress and radiation (12).

The significance of IL-11 with respect to its clinical implications in the cardiovascular field has not been well studied. Recently, we showed that the IL-11 receptor is expressed in cardiomyocytes and that IL-11 stimulation induces STAT3 activation (3), which regulates various cellular processes including cell growth and apoptosis. We also demonstrated that pre-ischemic administration of IL-11 offers protection against IR injury in murine hearts through a preconditioning effect (3). Notably, administration of IL-11 after inducing myocardial infarction, which was generated by coronary ligation, ameliorated the post-infarct cardiac dysfunction. The post-ischemic treatment with IL11 prevented cardiac fibrosis, reduced the frequency of cell death, and promoted angiogenesis $(4,5)$.

The present study showed that IL-11 alleviated the destined functional impairment of the heart after prolonged ischemia and reperfusion in a donor heart model. The mechanism underlying this effect may be attributed to the stimulation of STAT3 activated by IL-11. As described previously (13), cardiac myocytes release large quantities of IL-6 family cytokines, including IL-11, in conditions of pathological stress. These cytokines activate a common signaling pathway, the JAK/STAT pathway, through their unique receptor system. In response to cytokine engagements to their specific receptors, which invariably interact with a cytokine-specific transmembrane protein (gp130) that triggers Janus kinases (JAK) to phosphorylate a family of transcription factors (STAT), various intracellular 


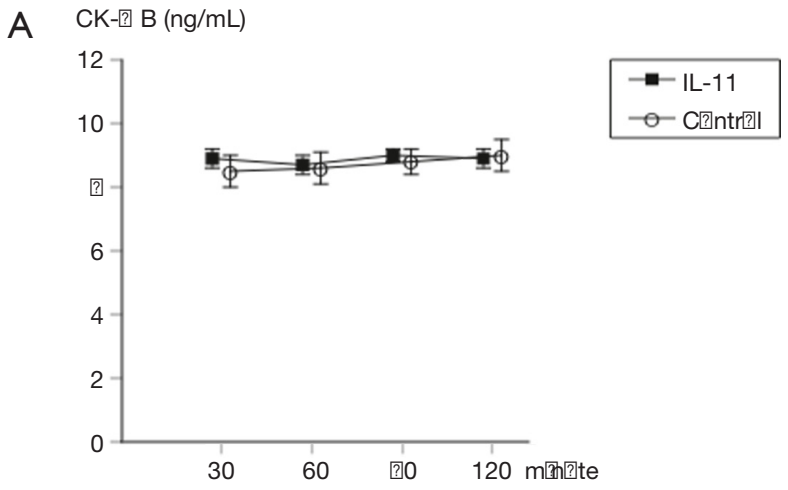

B

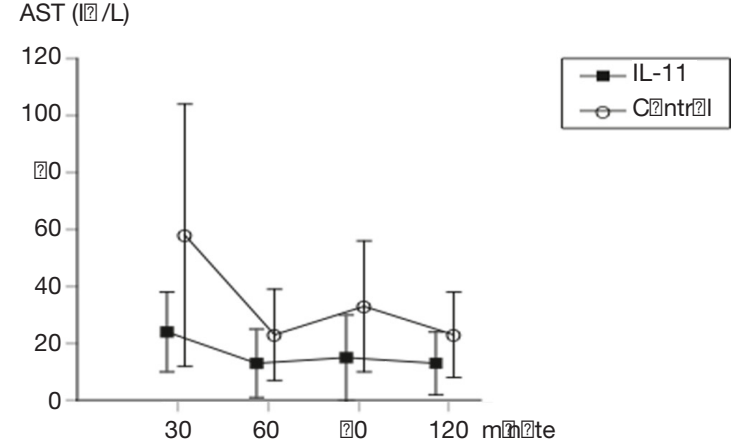

Figure 2 Comparison of cardiac biomarkers. There were no significant differences in CK-MB (A) and AST (B) measured at each reperfusion interval between the two groups. Data are shown as mean \pm standard deviation. The comparison between both groups was performed using an unpaired $t$-test. IL-11, interleukin-11; CK-MB, Creatine kinase MB isoenzyme; AST, aspartate aminotransferase.

signals are generated, causing the cell to transform. STAT3 in particular mediates a signal transduction that promotes cellular growth and reverses apoptosis, and thus plays a key role in neovascularization and cellular survival. Studies have shown that deletion of STAT3 in mice causes cardiomyopathy (14), and in patients with the disease, the level of STAT3 in the myocardia was significantly low (15). All these suggest that activating the JAK/STAT3 pathway may be the key to preventing the onset of heart failure.

In this study, we demonstrated that IL-11 improved the contractile function of the heart and reduced the number of apoptotic cells. However, we are uncertain if these results were directly associated with apoptosis. We did see that the hearts that performed worse had more apoptotic cells, but the quantity of the cells was small and insufficient to explain the significant difference in the contractile performances. Interestingly, none of the isolated hearts showed necrosis,

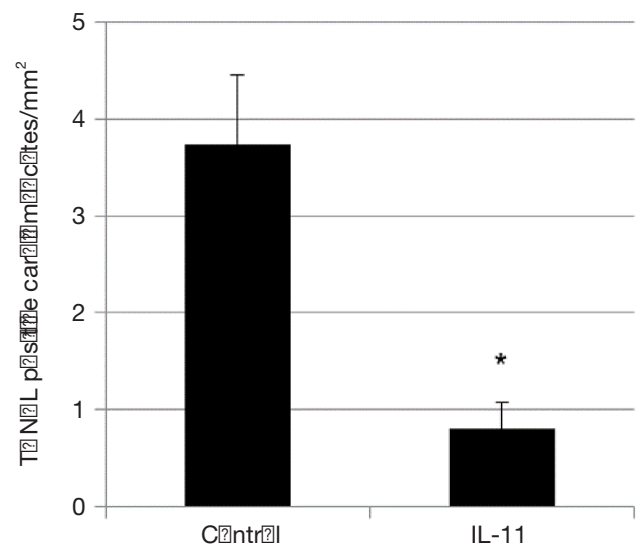

Figure 3 Comparison of the number of apoptotic cardiomyocytes. The number of TUNEL-labeled cardiomyocytes was significantly smaller in the IL-11 group than the control $(0.8 \pm 0.3$ vs. $3.7 \pm 0.7 / \mathrm{mm}^{2}, 5$ rats each, $\left.\mathrm{P}=0.0002\right)$. Data are shown as mean \pm standard deviation. ${ }^{*}, \mathrm{P}<0.05$ by unpaired $t$-test. IL-11, interleukin-11; TUNEL, terminal deoxynucleotide transferasemediated dUTP nick-end labeling.

as evidenced by the results of our TTC staining and the cardiac biomarkers collected from the coronary effluents. Previous studies have suggested that apoptosis is triggered at the time of reperfusion and does not manifest during the ischemic period, given that apoptosis is energy-dependent and ATP levels are depleted during ischemia $(16,17)$. These studies imply that the apoptotic component of myocardial dysfunction after IR injury reflects the activation of multiple genetic pathways and generally requires a time lag, usually 24 hours after reperfusion, for it to be apparent. In this context, the 2-hour reperfusion time used for evaluation in this study may be too short to rationalize apoptosis as a primary factor for the significant difference in the functional recovery of the two groups. We therefore suspect that our results were also attributed to a different pathway existing upstream of apoptosis. It has been shown that STAT3 not only influences the apoptotic pathway but also reduces reactive oxygen species (ROS); the JAK/ STAT3 pathway upregulates the transcription of manganese superoxide dismutase and metallothionein, both of which are known ROS scavengers (13). ROS act as mediators for post-ischemic myocardial stunning, and studies have shown that reperfusion of the ischemic hearts with ROS scavengers improves functional recovery and reduces the occurrence of IR-induced arrhythmias (18). We therefore hypothesize that IL-11 can attenuate myocardial stunning and engender 
quick recovery of the myocardium by eliciting ROS scavenging through STAT3 activation. Further studies, however, are warranted to confirm this.

This study has several limitations, including the study size and the determination of cold ischemic time, both of which were based on a small number of animals. The most important limitation, however, would be the method of IL11 administration. Optimization of the dose and timing of IL-11 administration was not considered in this study but is an important topic as it may influence the outcome. What formed the basis of the present dosing strategy was the results of our previous IL-11 studies (3-5). In a study using a mouse myocardial IR injury model, the animals pretreated with $8 \mu \mathrm{g} / \mathrm{kg}$ of IL-11 had a significantly reduced infarct size (4). In other studies using larger animals (murine and canine) that received IL-11 after induction of ischemia, we found that IL-11 ameliorated cardiac dysfunction and reduced fibrosis in a dose-dependent manner; a dose of $16 \mu \mathrm{g} / \mathrm{kg}$ or more significantly preserved cardiac function (19). Both pre-ischemic and post-ischemic IL11 administration have bestowed favorable outcomes, thus encouraging us to apply both in the present study (12 and $18 \mu \mathrm{g} / \mathrm{kg}$, respectively). In the clinical setting, recombinant human IL-11 is used for treatment of post-chemotherapy thrombocytopenia in which the therapeutic dose may be increased up to $50 \mu \mathrm{g} / \mathrm{kg}$ per day without adverse effects $(3,4)$. In a recent report using IL-11 to treat acute myocardial infarction, IL-11 was administered at a dose of $25 \mu \mathrm{g} / \mathrm{kg}$ and no side effects were observed (20). The reported side effects of IL-11 include edema, facial flushing, and conjunctival hyperemia (21). Nevertheless, optimization of the protocol is mandatory and will be addressed in future studies.

In conclusion, administration of IL-11 significantly protected the heart from IR injury following prolonged global hypothermia. This effect may be attributed to the direct transduction of cardioprotective signals mediated by STAT3 following increased IL-11 engagement on affected cells, causing reduced frequency of cell death and possibly alleviating the intensity of myocardial stunning. Although we are uncertain if apoptosis had direct association with the functional recovery of the heart in our IR model, the fact that IL-11 did influence the apoptotic pathway, as shown by the decreased incidence of the phenomenon, does imply that IL-11 may also confer substantial benefit in the later phase of recovery. If translated to a clinical setting, we may not only experience a quick recovery after prolonged ischemia but also a decreased incidence of graft failures after cardiac transplantation. From this perspective, the role of IL-11 therapy as a cardioprotective adjunct, together with its clinically recognized and safety-assured pharmacological profile, offers enormous potential and considerable advantages over other similar agents. However, further studies will be needed to ascertain the clinical relevance of these findings.

\section{Acknowledgements}

This study could not have been performed without the constant backup from Dr. Michiko Watanabe, Dr. Kazuyoshi Fukazawa of Chiba University Hospital Department of Cardiovascular Surgery. Deep appreciation is extended to Ms. Yuko Ohta for her contribution and technical assistance during the experiment. We also thank Mrs. Megumi Hosaka and Ms. Yukari Sakurai for their support in the preparation of this manuscript.

Funding: This work was supported by JSPS KAKENHI Grant Number JP23592027.

\section{Footnote}

Conflicts of Interest: The authors have no conflicts of interest to declare.

Ethical Statement: The study was approved by the institutional committee.

\section{References}

1. Keck BM, White R, Breen TJ, et al. Thoracic organ transplants in the United States: a report from the UNOS/ ISHLT Scientific Registry for Organ Transplants. United Network for Organ Sharing. International Society for Heart and Lung Transplantation. Clin Transpl 1994:37-46.

2. Du X, Williams DA. Interleukin-11: review of molecular, cell biology, and clinical use. Blood 1997;89:3897-908.

3. Kimura R, Maeda M, Arita A, et al. Identification of cardiac myocytes as the target of interleukin 11, a cardioprotective cytokine. Cytokine 2007;38:107-15.

4. Obana M, Maeda M, Takeda K, et al. Therapeutic activation of signal transducer and activator of transcription 3 by interleukin- 11 ameliorates cardiac fibrosis after myocardial infarction. Circulation 2010;121:684-91.

5. Obana M, Miyamoto K, Murasawa S, et al. Therapeutic administration of IL-11 exhibits the postconditioning effects against ischemia-reperfusion injury via 
STAT3 in the heart. Am J Physiol Heart Circ Physiol 2012;303:H569-77.

6. Cairo MS, Plunkett JM, Schendel P, et al. The combined effects of interleukin-11, stem cell factor, and granulocyte colony-stimulating factor on newborn rat hematopoiesis: significant enhancement of the absolute neutrophil count. Exp Hematol 1994;22:1118-23.

7. Kobayashi S, Teramura M, Oshimi K, et al. Interleukin-11. Leuk Lymphoma 1994;15:45-9.

8. Gordon MS, McCaskill-Stevens WJ, Battiato LA, et al. A phase I trial of recombinant human interleukin-11 (neumega rhIL-11 growth factor) in women with breast cancer receiving chemotherapy. Blood 1996;87:3615-24.

9. Kaye JA. FDA licensure of NEUMEGA to prevent severe chemotherapy-induced thrombocytopenia. Stem Cells 1998;16 Suppl 2:207-23.

10. Takeuchi $Y$, Watanabe $S$, Ishii $G$, et al. Interleukin-11 as a stimulatory factor for bone formation prevents bone loss with advancing age in mice. J Biol Chem 2002;277:49011-8.

11. Sands BE, Winston BD, Salzberg B, et al. Randomized, controlled trial of recombinant human interleukin-11 in patients with active Crohn's disease. Aliment Pharmacol Ther 2002;16:399-406.

12. Du X, Liu Q, Yang Z, et al. Protective effects of interleukin-11 in a murine model of ischemic bowel necrosis. Am J Physiol 1997;272:G545-52.

13. Fujio $Y$, Maeda M, Mohri T, et al. Glycoprotein 130 cytokine signal as a therapeutic target against cardiovascular diseases. J Pharmacol Sci 2011;117:213-22.

14. Camporeale A, Marino F, Papageorgiou A, et al. STAT3

Cite this article as: Tamura $\mathrm{Y}$, Kohno H, Mohri T, Fujio Y, Matsumiya G. The cardioprotective effect of interleukin-11 against ischemia-reperfusion injury in a heart donor model. Ann Cardiothorac Surg 2018;7(1):99-105. doi: 10.21037/ acs.2017.09.11 activity is necessary and sufficient for the development of immune-mediated myocarditis in mice and promotes progression to dilated cardiomyopathy. EMBO Mol Med 2013;5:572-90.

15. Podewski EK, Hilfiker-Kleiner D, Hilfiker A, et al. Alterations in Janus kinase (JAK)-signal transducers and activators of transcription (STAT) signaling in patients with end-stage dilated cardiomyopathy. Circulation 2003;107:798-802.

16. Zhao ZQ, Nakamura M, Wang NP, et al. Reperfusion induces myocardial apoptotic cell death. Cardiovasc Res 2000;45:651-60.

17. Leist M, Single B, Castoldi AF, et al. Intracellular adenosine triphosphate (ATP) concentration: a switch in the decision between apoptosis and necrosis. J Exp Med 1997;185:1481-6.

18. Kevin LG, Novalija E, Stowe DF. Reactive oxygen species as mediators of cardiac injury and protection: the relevance to anesthesia practice. Anesth Analg 2005;101:1275-87.

19. Takeda K, Matsumiya G, Miyagawa S, et al. Abstract 3031: Interleukin-11 Attenuates Infarct Size and Improves Ventricular Function in a Canine Heart Model of Ischemia/Reperfusion Injury. Circulation 2009;120:S737.

20. Nakagawa M, Owada Y, Izumi $Y$, et al. Four cases of investigational therapy with interleukin-11 against acute myocardial infarction. Heart Vessels 2016;31:1574-8.

21. Smith JW 2nd. Tolerability and side-effect profile of rhIL11. Oncology (Williston Park) 2000;14:41-7. 